\title{
Recent Improvements in the Detection of Supernovae with the IceCube Observatory
}

\section{The IceCube Collaboration ${ }^{\dagger}$,}

${ }^{\dagger}$ http://icecube.wisc.edu/collaboration/authors/icrc15_icecube

E-mail: Volker.Baum@icecube.wisc.edu

\begin{abstract}
IceCube monitors one cubic kilometer of deep Antarctic ice with a lattice of 5160 optical sensors in search of neutrino signals from supernovae. Charge secondaries arising from neutrino interactions in the ice produce Cherenkov photons that are registered by photomultipliers in the sensors. Due to subfreezing ice temperatures, their dark rates are particularly low. Therefore a collective rate enhancement introduced by interacting neutrinos in all photomultipliers provides excellent sensitivity for core collapse supernovae of galactic origin. A detailed understanding of the characteristics and temporal changes of the dark rate background has been achieved and cosmic ray induced muons, responsible for the majority of fake supernova candidate triggers, are rejected in real time. An addition to the standard data acquisition allows IceCube to buffer all registered photons in the detector in case of a serious alert. By analyzing such data, a precision determination of the burst onset time and the characteristics of rapidly varying fluxes, as well as estimates of the average neutrino energies and - for supernovae ending in a black hole - of the burst direction may be obtained. Such data are also crucial to characterize details of the noise behavior and of the atmospheric muon background.
\end{abstract}

Corresponding authors: V. Baum*1, B. Eberhardt ${ }^{1}$, A. Fritz ${ }^{1}$, D. Heereman ${ }^{2}$, B. Riedel ${ }^{3}$

${ }^{1}$ Institute of Physics, University of Mainz

${ }^{2}$ Interuniversity Institute for High Energies, Université Libre de Bruxelles, Belgium

${ }^{3}$ Department of Physics, University of Alberta, Canada

The 34th International Cosmic Ray Conference,

30 July- 6 August, 2015

The Hague, The Netherlands

*Speaker. 


\section{Introduction}

IceCube, a grid of 5160 photo sensors imbedded in the ice of the Antarctic glacier, is uniquely suited to monitor our Galaxy for supernovae due to its $1 \mathrm{~km}^{3}$ size and its location. In the inert and $-43^{\circ} \mathrm{C}$ to $-20^{\circ} \mathrm{C}$ cold ice, IceCube's photomultiplier noise rates average around 540 Hz. At depths between $1450-2450 \mathrm{~m}$, the detector is partly shielded from cosmic ray induced muons. The inverse beta process $\bar{v}_{\mathrm{e}}+\mathrm{p} \rightarrow \mathrm{e}^{+}+\mathrm{n}$ dominates supernova neutrino interactions with $\mathscr{O}(10 \mathrm{MeV})$ energy in ice, leading to positron tracks of about $0.6 \mathrm{~cm} \cdot E_{V} / \mathrm{MeV}$ length which radiate $178 \cdot E_{\mathrm{e}^{+}} / \mathrm{MeV}$ Cherenkov photons in the $300-600 \mathrm{~nm}$ wavelength range. From the approximate $E_{v}^{2}$ dependence of the cross section and the linear energy dependence of the track length, the light yield per neutrino roughly scales with $E_{v}^{3}$. With absorption lengths exceeding $100 \mathrm{~m}$, photons travel long distances in the ice so that each DOM effectively monitors several hundred cubic meters of ice. Typically, only a single photon from each interaction reaches one of the photomultipliers that are vertically (horizontally) separated by roughly $17 \mathrm{~m}(125 \mathrm{~m})$. The DeepCore subdetector, equipped with a denser array of high efficiency photomultipliers, provides higher detection and coincidence probabilities.

Although the rate increase in individual light sensors is not statistically significant, the effect will be clearly seen once the rise is considered collectively over many sensors. IceCube is the most precise detector for analyzing the neutrino light curve of close supernovae [1]. Since 2009, IceCube has been sending real-time data grams to the Supernova Early Warning System (SNEWS) [2] when detecting supernova candidate events. The supernova data acquisition is based on count rates of individual optical modules stored in $1.6384 \mathrm{~ms}$ time bins. The analysis framework calculates the average dark noise rate deviation $\Delta \mu$ normalized to its own width $\sigma_{\Delta_{\mu}}$ and calculates for each candidate the significance $\xi=\Delta \mu / \sigma_{\Delta_{\mu}}$. In addition, all time-stamped hits in the detector are buffered and extracted for supernova candidate triggers (hitspooling) [3].

In this paper, we first characterize the effect of the atmospheric muon background and introduce a subtraction method for the associated hits. We then discuss in detail the properties of the photomultiplier dark noise on top of which the supernova neutrino signal would be observed and we will finally quantify the directional resolution that can be obtained for a class of supernovae that abruptly form a black hole.

\section{Improved significance calculation by correcting for atmospheric muon hits}

Atmospheric muons require an energy of $\approx 400 \mathrm{GeV}$ at the surface to reach the detector (see Fig. 1, left) and $\approx 550 \mathrm{GeV}$ to trigger the IceCube 8-fold majority trigger; hits from muon tracks that fail the trigger requirements are mostly found in the upper detector layers or are corner clippers (see Fig. 1, right). Dust layers in the ice, particularly the one $\approx 100 \mathrm{~m}$ below the detector center, absorb light. This effect and the limited muon range lead to a depth-dependent atmospheric muon induced rate between $\approx 3 \mathrm{~Hz}$ and $\approx 30 \mathrm{~Hz}$. While the fraction of such hits in each DOM is small compared to its dark rate, the effect on the summed hits as well as on the significance $\xi$ to observe a rate excess in all optical modules is much more apparent [4]. The reason is that muons lead to space and time correlated hits such that the optical sensor rates are not statistically independent and 

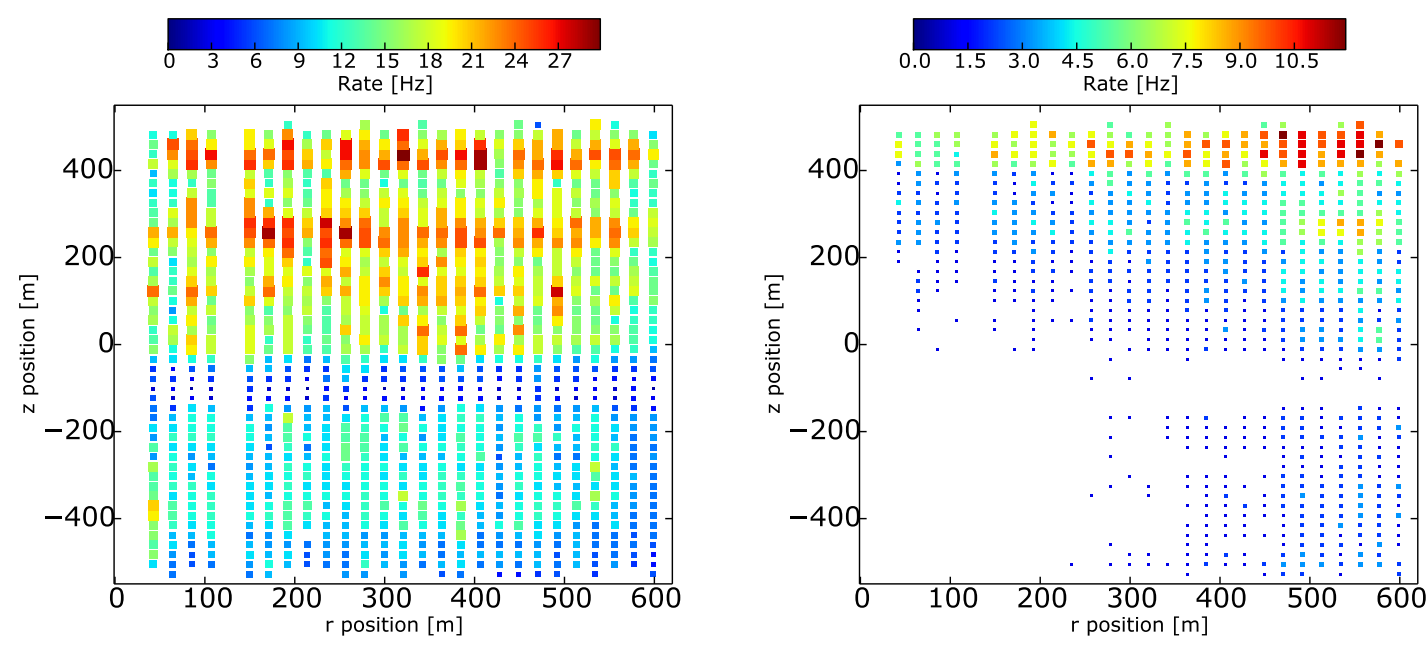

Figure 1: Simulated muon induced hit rates as function of horizontal position and radius w.r.t. to the detector center at $1950 \mathrm{~m}$ depth. Left: all hits associated with muons triggering the detector. Right: hits associated with muons not meeting any trigger condition.
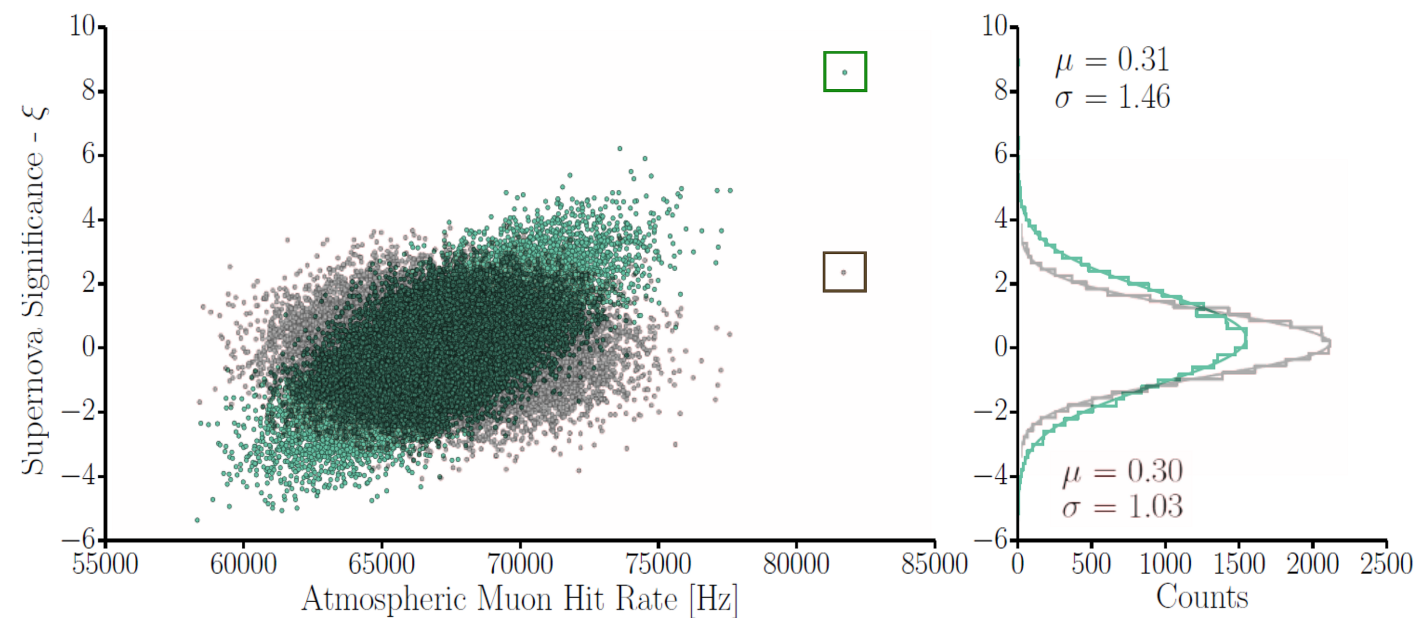

Figure 2: Correlation of the atmospheric muon induced hit rate with the significance $\xi$ (green). The application of the decorrelation discussed in the text leads to the gray distribution. The projections on the right hand side demonstrate the sharpening of the significance distribution after decorrelation. During this particular run, the highest significant candidate so far was observed (marked by a green square). After decorrelation, the significance dropped (marked by a gray square) verifying that the candidate was due to an upward fluctuation in the number of atmospheric muon hits.

the law of large numbers is no longer applicable. In fact, the vast majority of false positive triggers are due to statistical agglomerations of atmospheric muon induced hits.

In case of an alert, the only independent quantity to check whether the event was real or spurious is the cosmic ray induced muon activity during the respective period. We demonstrated in an offline analysis [4] that the separation of signal from background can be considerably improved by subtracting hits associated to atmospheric muon induced tracks. The subtraction method fits a linear function to the correlation between the simple majority trigger hit rate $R_{\mu}^{\text {hit }}$ as a mea- 
sure for the atmospheric muon rate and the significance $\xi$ (see Fig. 2 for an example 8 hour run) and calculates a corrected significance $\xi^{\prime}=\xi-b \cdot R_{\mu}^{\text {hit }}-a$ from the resulting offset $a$ and slope $b$. This method lowers the significance of the sample alert from $\xi=8.59$ to $\xi^{\prime}=2.44$ and decreases the width of the significance distribution from 1.46 to 1.03 , close to the expectation of unity for uncorrelated noise. Until recently, such a study could only be performed offline with a few days delay due to limited satellite time availability and bandwidth. In order to perform an atmospheric muon correction in real time, the number of hits processed by IceCube's majority trigger is transmitted to the supernova data acquisition system. To guarantee a robust system and to limit the increase in processing time, the data are only processed once a predefined significance threshold $\xi_{\text {cut }}$ is crossed. The method reduces the number of fake alarms by almost three orders of magnitude when keeping the same alarm threshold as before. The additional memory and CPU requirements do not affect the stability of the system so that one may now lower the alarm thresholds. By applying a cut on $\xi>4.0$ before atmospheric muon subtraction one does already achieve a factor of $10^{-2}$ in background reduction compared to the original threshold of $\xi>7.65$ (see Fig. 3) which further improves to about $10^{-7}$ by switching to the combination $\xi>4.0, \xi^{\prime}>5$ 5.6. Using this combination we are also able to increase the SNEWS alarm efficiency for po-

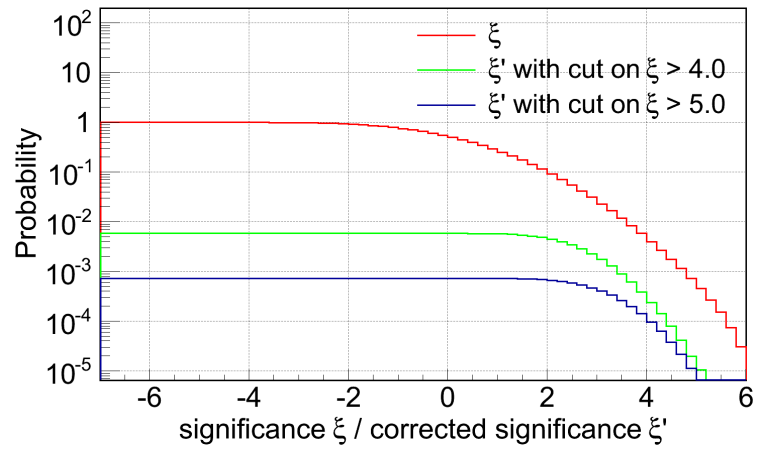

Figure 3: Cumulative probabilities to detect an event with significance $\xi$ (red) and to detect the corrected significance $\xi^{\prime}$ after additional cuts on $\xi$ (Cuts shown are $\xi>4.0$ and $\xi>5.0$ in green and blue.)

tential supernovae in the Large Magellanic Cloud from merely $12 \%$ to $82 \%$, while meeting the SNEWS requirement to sent alarms with a frequency of less than one alarm per 10 days.

\section{Long term behavior of DOM dark rates}

Over the course of the first three years of the completed detector (IC86-2011, IC86-2012, IC86-2013), the number of false positive supernova alerts increased by almost $50 \%$ with time. This came as a surprise as we did not expect the sensitivity to cosmic ray induced muons to increase while the detector configuration remained unchanged. As can be seen from Fig. 4, the average dark noise rate of the optical modules decreased with time, on average, by $3.5 \%$. This decrease led to an increased sensitivity to atmospheric muons of $\approx 2 \%$ and - as discussed above - to a disproportional increase in the false positive trigger rate. The decrease in the dark noise rate is correlated with the deployment date and the depth of the DOMs. It is particularly evident in the lower, warmer section of the detector, where the pressure is highest and the ice is under mechanical stress. While no definite source could be established, we suspect that the emission of light is caused by triboluminescence caused by the freezing of the ice in the bore holes. 


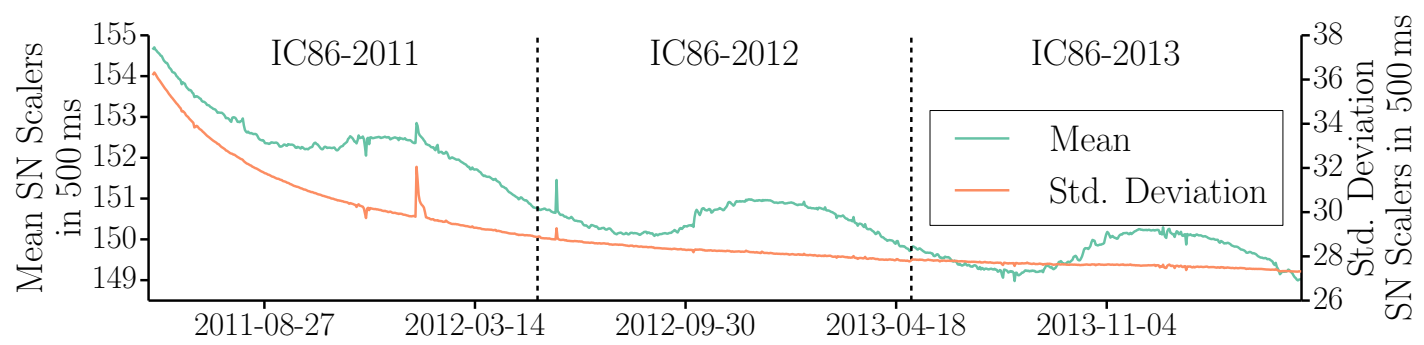

Figure 4: Temporal development of the mean SN scaler rate and standard deviation in $500 \mathrm{~ms}$ time bins after applying an artificial dead time of $250 \mu \mathrm{s}$. Both, seasonal varying influence of atmospheric muons and an overall decrease in the dark rate, are apparent in the mean rate (blue curve), while the standard deviation is rather insensitive to the influence of muons (orange curve). The rate spikes are detector related; in particular, the dark rate increased after light was emitted during the operation of a camera in the deep ice in early 2012.

\section{Exploiting detailed information from spooling all hits}

The buffered information from all photomultiplier hits in case of a trigger provides several advantages: on the one hand, the complete detector information is available for a subset of highly interesting events (such as supernova candidates or very high energetic events of astrophysical origin). On the other hand, these data - buffered at an early stage of the data acquisition system on the so-called string hubs - will be available in the unlikely case that the data acquisition may fail (i.e. in the case of an extremely close supernova which could exhaust the system). The automatized hitspooling system has been working reliably for several years, including the automatic transfer of data in case of significant alerts to the North. The data from these triggered events have been carefully studied and compared to the results from the standard supernova data acquisition. There is - in general - good agreement, considering that hitspooling has no artificial dead time applied to reduce non-Poissonian dark noise and when taking into account the larger uncertainty on the moving average noise determination when using the $90 \mathrm{~s}$ long hitspooling data fragment rather than the $10 \mathrm{~min}$ available in the scaler based data acquisition system. The detailed information available in the hitspooling data can be exploited for several purposes, some of which we will discuss below:

- basic studies of the non-Poissonian characteristics of dark noise at low temperatures,

- characterization of the dark noise for individual sensors to improve IceCube's Monte Carlo simulations,

- application of sophisticated techniques to identify noise hits or hits associated with atmospheric muons that do not trigger the IceCube majority trigger,

- determination of coincidence probabilities for the estimation of the average supernova neutrino energy [5], and

- triangulation of the supernova direction in the case that signals change abruptly.

Non-Poissonian noise characteristics: Hitspooling data were used to investigate the characteristics of correlated dark noise that rises at low temperatures and compare in-ice measurements with those taken in the laboratory. Figure 5 shows the IceCube dark rate as function of depth-dependent 
temperature at the PMT photo cathodes. Each data point represents the average of 12 optical module layers with DeepCore excluded; ref. [6] was used to translate the known depth into an ice temperature. Correlated noise, suspected to be due to glass fluorescence, comes in bursts, while uncorrelated noise follows a Poissonian distribution. By defining a burst as an uninterrupted sequence of $<3 \mathrm{~ms}$ intervals, the number of hits $L$ present in the burst, the frequency of burst $F_{L}$ with a given number of hits, the time between the last and the first hit in a burst $D_{L}$, and the inter-burst interval distribution were measured and compared to previous results by Meyer [7]. The average number of hits per burst rises roughly linearly from 3.3 hits at $-10{ }^{\circ} \mathrm{C}$ to 3.8 hits at $-33{ }^{\circ} \mathrm{C}$. Figures 6, left and right, show the corresponding distributions which are in general in agreement with the findings of [7]. For instance, the average burst duration $D_{L}$ follows Meyer's anticipation of a power law proportional to $L^{1 / 2}$ with the difference that we identify two separate regions for best fits.

\section{Improved muon identification and rejection: Sim-} ulation data show that $\sim 50 \%$ of all atmospheric muons crossing the detector are triggered. The remaining half leads to a factor of three fewer hits mostly at the top of the detector (see Fig.1, right); $26 \%$ of all subthreshold muons produce only a single hit in the detector. Removing these subthreshold muons hits may be achieved by restricting oneself to hits in the lower part of the detector or by using algorithms developed to identify hits clustering in time and space combined with an investigation of the multiplicity of track segments formed by each pair of hits in the cluster. With the second method, it is possible to identify up to $45 \%$ of all sub-threshold muons hits, limited only by the requirement of at least 4 hits to define a cluster in the algorithms.

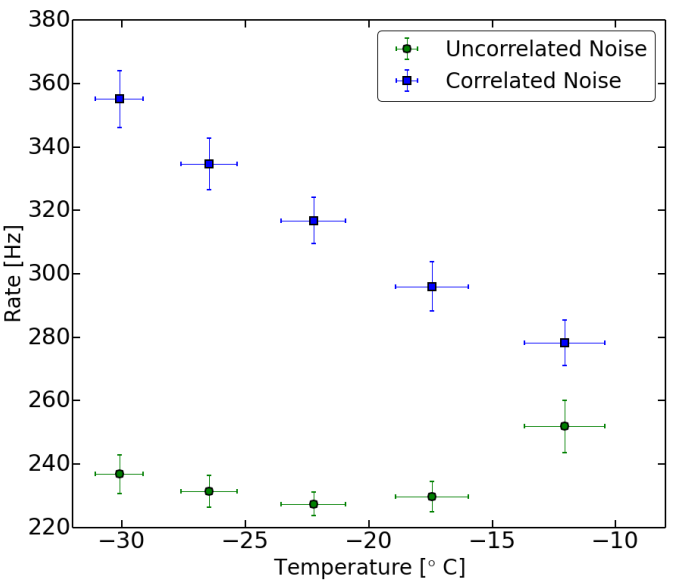

Figure 5: Non-Poissonian correlated and Poissonian uncorrelated components of IceCube's dark rate as function of the estimated temperature close to the PMT cathode.
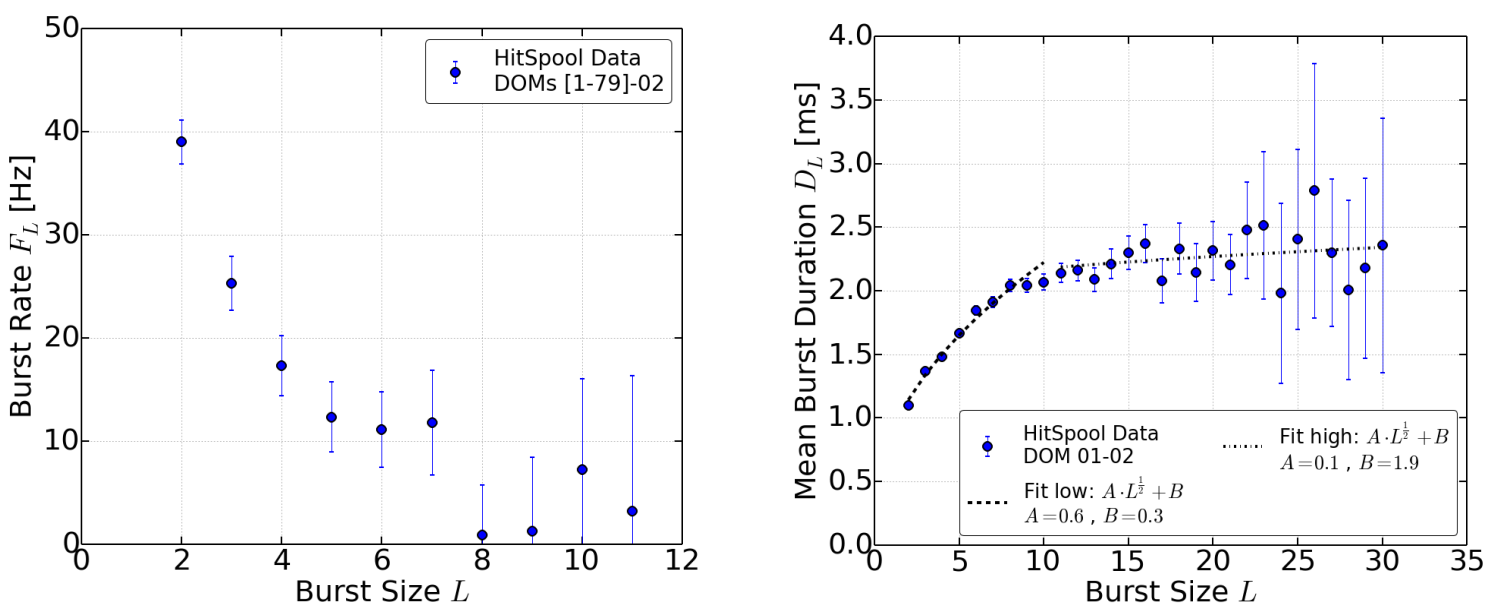

Figure 6: Burst rate $F_{L}$ (left) and average burst duration $D_{L}$ (right) as function of burst size $L$. 
Applying these techniques to experimental data results in a $\sim 3 \%$ decrease of the total hit rate and reduces the fraction of false positive SNEWS alarms by an additional $45 \%$. In the future, we will use this improvement in the determination of the average neutrino energy.

Triangulation of supernova direction: IceCube cannot identify individual neutrino interactions as usually only one photon is detected per interaction. In addition, the direction of the positron is essentially uncorrelated with the incoming neutrino direction for the dominant inverse beta decay interaction. Therefore it is a challenge to measure the supernova direction with IceCube. Triangulation with other detectors has been proposed $[8,9]$ as one possibility. Another option is to determine the direction from the temporal hit pattern seen in the cubic-kilometer detector when a neutrino wavefront changing its intensity abruptly. The detector crossing lasts only several microseconds, which sets the time-scale for abrupt flux change to be suitable.

One example is the formation of a black hole following a core collapse of a super-massive star. In case the protoneutron star forms a black hole instead of gradually cooling as a stable neutron star, the neutrino flux should cease almost immediately, once the Schwarzschild condition is met [10]. The neutrino fluxes may also gradually decrease as more and more matter in the star approaches the event horizon and the gravitational redshift becomes extremely strong [11] (see green curve in Fig. 7). In addition, the neutrinos follow a spectrum of energies, which - due to the non-vanishing neutrino masses - leads to a smearing of the time arrival distribution (see Fig. 7). Black holes forming core collapse supernovae have therefore been suggested as a way to determine the neutrino mass [10]. We first adopt the optimistic scenario that the smearing due to the black hole dynamics is negligible [12]. An unbinned likelihood analysis with an optimized minimizer is used to determine the direction from the timing pattern of the hit DOMs. Figure 8 shows the simulated resolution achieved as function of distance (left) and as function of neutrino masses (right). Obviously, reasonable directional resolution can be achieved only for close-by supernovae and low neutrino masses.

Using the assumption of a gradual decrease of neutrino luminosity [11] and numerically folding the distribution of [10] for the time-delay due to finite

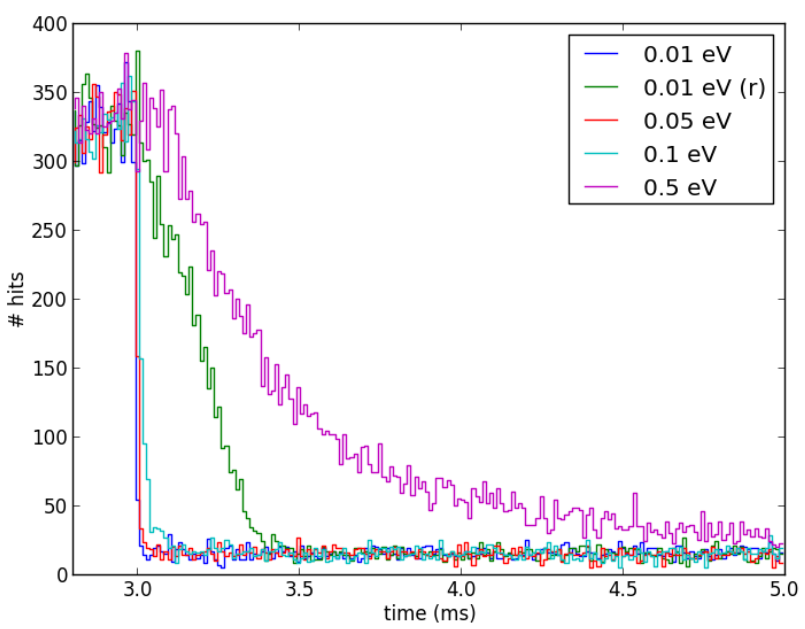

Figure 7: Effect of neutrino mass on the observed neutrino flux for abrupt black hole creation. The green line shows the effect of a gradual decrease of the neutrino luminosity $\left(\mathrm{m}_{v}=0.01 \mathrm{eV}\right)$. neutrino masses, one finds that directions can only be reconstructed for very close supernovae. 

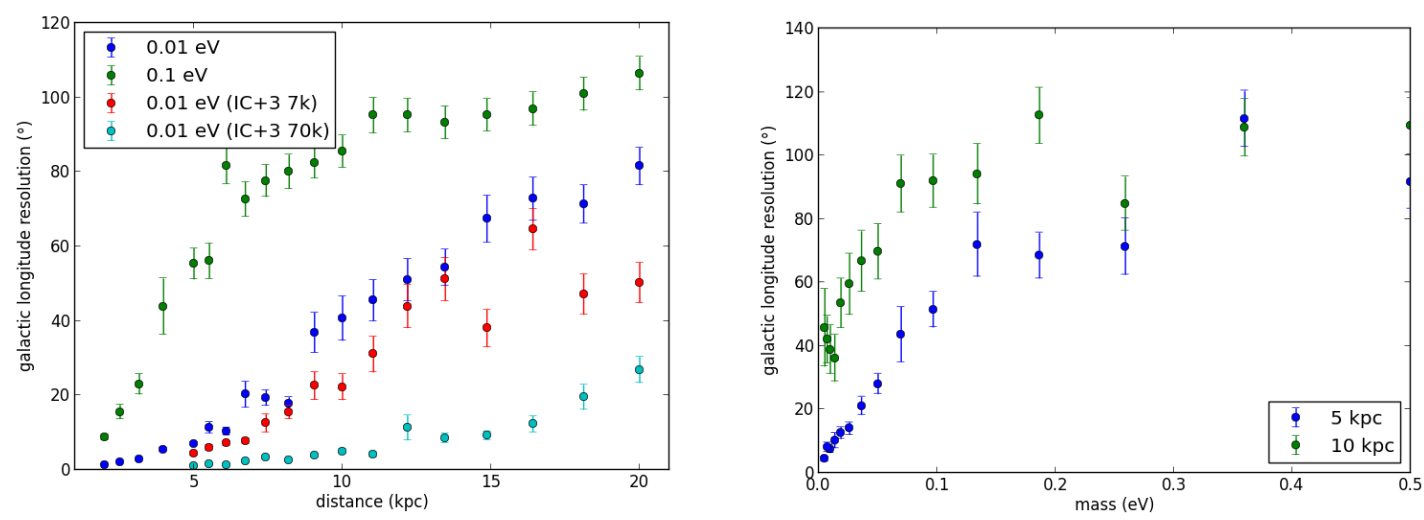

Figure 8: Left: Resolution in galactic longitude achievable by triangulation within IceCube's geometry for a supernova with abrupt black hole formation for two assumptions on the mass of the lightest neutrino $(0.01$ $\mathrm{eV}$ and $0.1 \mathrm{eV}$, respectively) as well as for a hypothetical detector extension with 3 additional IceCube strings deployed symmetrically around IceCube's center at 7 and $70 \mathrm{~km}$ distance, respectively. Right: Resolution in galactic longitude as function of the neutrino mass.

\section{Conclusions and Outlook}

The IceCube observatory provides the world's best statistical accuracy for the neutrino flux of supernovae in our galaxy with a round-the-clock up-time of close to $99 \%$. However, energies and directions of individual neutrinos can not be determined due to the optical sensor dark rates and - to a lesser extend - due to atmospheric muons passing the detector. Therefore it is very important to understand the dark rate and atmospheric muon characteristics. Neutrino energies and the supernova direction can be determined statistically when analyzing the timing information of all hits in the detector. In the future, the sensitivity to the absolute neutrino mass will be assessed, the energy determination will be improved, the idea of analyzing $\mathscr{O}(10) \mathrm{s}$ long bursts will be extended to shorter potential signals, and the hitspooling system will be used for other use cases, such as the estimate of hadronic energy in highly energetic events from the delayed neutron capture signal.

\section{References}

[1] R. Abbasi et al. (IceCube Collaboration), A\&A 535 (2011) A109.

[2] P. Antonioli et al., New J. Phys. 6 (2004) 114.

[3] D. Heereman, V. Baum, G. Kroll, and B. Riedel (IceCube Collaboration), contribution to the 33rd International Cosmic Ray Conference, arXiv:1309.7008 [astro-ph.HE]

[4] V. Baum, B. Eberhardt, G. Kroll, and B. Riedel (IceCube Collaboration), contribution to the 33rd International Cosmic Ray Conference, arXiv:1309.7008 [astro-ph.HE].

[5] R. Bruijn, Nucl. Phys. B Proc. Supp. 00 (2012) 13.

[6] P. Buford Price et al., Proceedings of the National Academy of Sciences 99 (2002) 12:7844.

[7] H.O. Meyer, EPL 89 (2010) 58001.

[8] R. Tomas, D. Semikoz, G.G. Raffelt, M. Kachelriess, and A.S. Dighe, Phys. Rev. D 68 (2003) 093013.

[9] T. Mühlbeier, H. Nunokawa and R. Zukanovich Funchal, Phys. Rev. D 88 (2013) 085010.

[10] J.F. Beacom, R.N. Boyd, and A. Mezzacappa, Phys. Rev. D 63 (2001) 073011.

[11] T.E. Baumgarte, S.L. Shapiro, S.A. Teukolsky, Astrophys.J. 458 (1996) 680.

[12] K. Sumiyoshi, S. Yamada and H. Suzuki, Astrop.J. 667 (2007) 382. 